\title{
A lower bound on the size of resolution proofs of the Ramsey theorem
}

\author{
Pavel Pudlák*
}

December 7, 2011

\begin{abstract}
We prove an exponential lower bound on the lengths of resolution proofs of propositions expressing the finite Ramsey theorem for pairs.
\end{abstract}

Assuming that $n \geq R(k)$, where $R(k)$ denotes the Ramsey number, the Ramsey theorem for pairs and two colors, $n \rightarrow(k)_{2}^{2}$, is presented by the following unsatisfiable set of clauses. The variables are $x_{i j}$, for $1 \leq i<j \leq n$. The clauses are $\bigvee_{i, j \in K} x_{i j}$ and $\bigvee_{i, j \in K} \neg x_{i j}$, for all sets $K \subseteq\{1, \ldots, n\},|K|=k$. The corresponding tautology will be denoted by $R A M(n, k)$.

The Ramsey theorem was proposed as a hard tautology by Krishnamurthy in [6]. He studied the tautology $R A M(R(k), k)$ and proved a lower bound $R(k) / 2$ on the width of resolution proofs (see also [5]). This implies an exponential lower bound on the tree-like resolution proofs. Krajíček proved an exponential lower bound on this tautology by reducing the proofs of the pigeonhole principle to it, [4]. The problem with this tautology is that we do not know what is $R(k)$. This prevent us from proving an upper bound on the proof complexity of this tautology. Therefore researchers focused on the tautology $R A M(n, k)$ for $k=\left\lfloor\frac{1}{2} \log n\right\rfloor$ (all $\log$ arithms are to the base 2 in this paper). This tautology is provable in a bounded depth Frege system, see [7, 4]. For this tautology, Krajíček proved an exponential lower bound on tree-like resolution proofs with conjunctions of logarithmic size, [3]. The complexity of unrestricted resolution proofs with conjunctions of logarithmic size proofs of $R A M\left(n,\left\lfloor\frac{1}{2} \log n\right\rfloor\right)$ is still an open problem. An exponential lower bound on such proofs would have interesting consequences in proof complexity and bounded arithmetic. In particular it would give a separation of the relativized theories $T_{2}^{2}$ and $T_{2}^{3}$ by a $\forall \Sigma_{1}^{b}$ sentence (see $[2,1])$. In this paper we prove an exponential lower bound on unrestricted resolution proofs.

Theorem 1 Resolution proofs of $\operatorname{RAM}\left(n,\left\lfloor\frac{1}{2} \log n\right\rfloor\right)$ have size at least $2^{n^{\frac{1}{4}-o(1)}}$.

*Institute of Mathematics, Academy of Sciences, Prague, and Institute of Theoretical Computer Science, Prague, e-mail: pudlak@math.cas.cz. Partially supported by Institutional Research Plan No. AV0Z10190503, project No. 1M0021620808 of MŠMT ČR and grant IAA100190902 of GA AV ČR. 
Proof. We will use the following bound for the sum of Bernoulli variables $X=\sum_{i=1}^{r} X_{i}$ with $\operatorname{Pr}\left(X_{i}=1\right)=q$.

$$
\operatorname{Pr}(X \geq c r) \leq q^{c r} 2^{H(c) r},
$$

where $H$ is the entropy function, which follows from $\left(\begin{array}{c}r \\ c r\end{array}\right) \leq 2^{H(c) r}$.

Let $\delta>0$. We will prove a lower bound $2^{\Omega\left(n^{\frac{1}{4}-\delta}\right)}$. Let $k=\left\lfloor\frac{1}{2} \log n\right\rfloor$ and $m=\left\lfloor n^{\frac{1}{4}-\delta}\right\rfloor$. In the rest of the proof we will ignore rounding. $\varepsilon$ and $p$ will be sufficiently small constant whose values will be determined later.

Let $\rho$ be the random restriction that sets $x_{i j}$ to 0 with probability $\frac{p}{2}, x_{i j}$ to 1 with probability $\frac{p}{2}$ and leaves $x_{i j}$ free with probability $1-p$. Let a proof $P$ be given and let $S$ be its size. After hitting $P$ by $\rho$, some clauses become true and we delete them. The others may have reduced length, because some literals become false. We will denote by $P_{\rho}$ the reduced proof. The probability that $P_{\rho}$ contains a clause of length $>\frac{m}{2}$ is less than

$$
S\left(1-\frac{p}{2}\right)^{\frac{m}{2}}=S \cdot 2^{\log \left(1-\frac{p}{2}\right) \frac{1}{2} n^{\frac{1}{4}-\delta}} .
$$

Hence, if $S<2^{-\log \left(1-\frac{p}{2}\right) \frac{1}{2} n^{\frac{1}{4}-\delta}-1}$, the probability is $<\frac{1}{2}$. We will assume this and show a contradiction.

Consider an initial clause. The probability that $\rho$ sets at least $\varepsilon\left(\begin{array}{l}k \\ 2\end{array}\right)$ literals of the clause is at most

$$
\left(\frac{p}{2}\right)^{\varepsilon}\left(\begin{array}{l}
k \\
2
\end{array}\right) 2^{H(\varepsilon)\left(\begin{array}{l}
k \\
2
\end{array}\right)}=2^{\left(\log \frac{p}{2} \cdot \varepsilon+H(\varepsilon)\right)\left(\begin{array}{l}
k \\
2
\end{array}\right)} \leq 2^{\frac{1}{8}\left(\log \frac{p}{2} \cdot \varepsilon+H(\varepsilon)+o(1)\right)(\log n)^{2}} .
$$

Hence the probability that this happens for at least one initial clause is at most

$$
\begin{array}{r}
2^{\frac{1}{8}\left(\log \frac{p}{2} \cdot \varepsilon+H(\varepsilon)+o(1)\right)(\log n)^{2}} \cdot 2\left(\begin{array}{l}
n \\
k
\end{array}\right) \leq \\
2^{\frac{1}{8}\left(\log \frac{p}{2} \cdot \varepsilon+H(\varepsilon)+o(1)\right)(\log n)^{2}} n^{\frac{1}{2} \log n}= \\
2^{\frac{1}{8}\left(\log \frac{p}{2} \cdot \varepsilon+H(\varepsilon)+\frac{1}{2}+o(1)\right)(\log n)^{2}} .
\end{array}
$$

If $p$ is sufficiently small w.r.t. $\varepsilon$, then the term $\log \frac{p}{2} \cdot \varepsilon+H(\varepsilon)+\frac{1}{2}+o(1)$ is negative for large $n$. Hence, for such a $p$ and large $n$ the probability is $<\frac{1}{2}$.

Thus there exists $\rho$ such that in the proof $P_{\rho}$

1. every clause has length at most $m / 2$;

2. every initial clause has at least $(1-\varepsilon)\left(\begin{array}{l}k \\ 2\end{array}\right)$ variables.

Following an idea of Krajíček [3], we will use a random graph $G$ on $m$ vertices to show that such a proof does not exist. While Krajíček only needed that $G$ does not have a homogeneous set of size $k$, we will need more: the number of edges on every subset of size $k$ is strictly between $\varepsilon\left(\begin{array}{l}k \\ 2\end{array}\right)$ and $(1-\varepsilon)\left(\begin{array}{l}k \\ 2\end{array}\right)$. (This is why we need $m$ larger than $n^{\frac{1}{4}}$.) The probability that this condition fails for one fixed set of size $k$ is at most

$$
2 \cdot 2^{-(1-\varepsilon)\left(\begin{array}{c}
k \\
2
\end{array}\right)}\left(\begin{array}{c}
\left(\begin{array}{c}
k \\
2
\end{array}\right) \\
\varepsilon\left(\begin{array}{l}
k \\
2
\end{array}\right)
\end{array}\right) \leq 2 \cdot 2^{(-1+\varepsilon+H(\varepsilon))\left(\begin{array}{l}
k \\
2
\end{array}\right)}=2^{(-1+\varepsilon+H(\varepsilon)+o(1)) \frac{(\log n)^{2}}{8}} .
$$


The probability that there exists a set of size $k$ for which it fails is at most

$$
\begin{array}{r}
2^{(-1+\varepsilon+H(\varepsilon)+o(1)) \frac{(\log n)^{2}}{8}} \cdot\left(\begin{array}{c}
m \\
k
\end{array}\right) \leq \\
2^{(-1+\varepsilon+H(\varepsilon)+o(1)) \frac{(\log n)^{2}}{8}} \cdot m^{k} \leq \\
2^{\frac{-1+\varepsilon+H(\varepsilon)+o(1)}{8}(\log n)^{2}+\frac{1}{2} \log n \cdot\left(\frac{1}{4}-\delta\right) \log n}= \\
2^{\frac{\varepsilon+H(\varepsilon)-4 \delta+o(1)}{8}(\log n)^{2}} .
\end{array}
$$

Hence if we choose $\varepsilon>0$ so that $\varepsilon+H(\varepsilon)<4 \delta$, the exponent will be negative for sufficiently large $n$. Thus we obtain the auxiliary graphs.

Now, as in Krajíček's proof, construct a path in $P_{\rho}$ from the empty clause to an initial clause such that for every clause $C$ on the path the following condition is satisfied. There exists a bijection between the indices of the variables of $C$ and vertices of the graph $G$ such that if $x_{i j}$ (or $\neg x_{i j}$ ) is a literal in $C$ and $u, v$ are vertices corresponding to $i, j$, then $(u, v)$ is not an edge (respectively is an edge in $G$ ). We can construct this path, because every clause has at most $m / 2$ literals. However the latter condition cannot be satisfied by the initial clauses, because each initial clause has at least $(1-\varepsilon)\left(\begin{array}{l}k \\ 2\end{array}\right)$ literals of the same kind, but in $G$ there are less than $(1-\varepsilon)\left(\begin{array}{l}k \\ 2\end{array}\right)$ pairs $(u, v)$ of the same kind (edges or non-edges) on every $k$-element subset. This contradiction finishes the proof.

\section{References}

[1] K. Aehlig, A. Beckmann, A remark on the induction needed to prove the Ramsey principle. 2006, unpublished.

[2] M. Chiari and J. Krajíček, Lifting independence results in bounded arithmetic, Archive for Mathematical Logic, 38(2), (1999), pp. 123-138.

[3] J. Krajíček, On the weak pigeonhole principle, Fundamenta Mathematicae, 170(1-3), (2001), pp. 123-140.

[4] J. Krajíček, A note on propositional proof complexity of some Ramsey-type statements, Archive for Mathematical Logic, 50(1-2), (2011), pp. 245-255.

[5] B. Krishnamurthy and R.N. Moll, Examples of hard tautologies in the propositional calculus. In: Proc. 13th ACM Symposium on Theory of Computing (1981), pp. 28-37.

[6] B. Krishnamurthy, Examples of hard tautologies and worst-case complexity results. Ph.D. Thesis, Univ. of Massachusetts, 1981.

[7] P. Pudlák: Ramsey's Theorem in Bounded Arithmetic. In: Proc. Computer Science Logic'90, eds. E. Borger, H. Kleine Buning, M.M. Richter, W. Schonfeld, LNCS 533, Springer-Verlag, 1991, pp. 308-317. 